\title{
Dust Emission from the Perseus Molecular Cloud
}

\section{Citation}

Schnee, S., J. Li, A. A. Goodman, and A. I. Sargent. 2008. “Dust Emission from the Perseus

Molecular Cloud." The Astrophysical Journal 684 (2): 1228-39. https://doi.org/10.1086/590375.

\section{Permanent link}

http://nrs.harvard.edu/urn-3:HUL.InstRepos:41397379

\section{Terms of Use}

This article was downloaded from Harvard University's DASH repository, and is made available under the terms and conditions applicable to Other Posted Material, as set forth at http:// nrs.harvard.edu/urn-3:HUL.InstRepos:dash.current.terms-of-use\#LAA

\section{Share Your Story}

The Harvard community has made this article openly available.

Please share how this access benefits you. Submit a story.

Accessibility 


\title{
DUST EMISSION FROM THE PERSEUS MOLECULAR CLOUD
}

\author{
S. Schnee, ${ }^{1}$ J. Li, ${ }^{2}$ A. A. Goodman, ${ }^{2}$ and A. I. Sargent ${ }^{1}$ \\ Received 2007 April 19; accepted 2008 May 19
}

\begin{abstract}
Using far-infrared emission maps taken by IRAS and Spitzer and a near-infrared extinction map derived from 2MASS data, we have made dust temperature and column density maps of the Perseus molecular cloud. We show that the emission from transiently heated very small grains (VSGs) and the big grain dust emissivity vary as a function of extinction and dust temperature, with higher dust emissivities for colder grains. This variable emissivity cannot be explained by temperature gradients along the line of sight or by noise in the emission maps, but it is consistent with grain growth in the higher density and lower temperature regions. By accounting for the variations in the dust emissivity and VSG emission, we are able to map the temperature and column density of a nearby molecular cloud with better accuracy than has previously been possible.
\end{abstract}

Subject headings: dust, extinction - ISM: clouds - surveys

Online material: color figures

\section{INTRODUCTION}

Far-infrared and submillimeter continuum radiation from interstellar clouds is typically attributed mostly to "dust." In truth, however, even the contributions from "dust" come from multiple populations of grain types, which are each at a mixture of temperatures. Along any given line of sight, there may be random or systematic variations both in the admixture of grain types and in their temperature, and those variations may or may not be correlated. Historically, researchers have used more and more complicated procedures to account for dust emission and estimate column density.

The simplest way to infer column density from "thermal" dust emission is to assume a single grain type, radiating with a single efficiency (opacity), at a single temperature. In that case, flux at any wavelength can be converted to a column density, with the assumption of a dust temperature. The next most sophisticated approach is to use observations at two wavelengths and to infer a color temperature by assuming that the dust emission comes from a modified blackbody, which has a wavelength-dependent emissivity that one takes as given (e.g., Wood et al. 1994).

With observations at three wavelengths, it should be possible to also constrain the dust emissivity $\left(\kappa_{\lambda}\right)$, if we assume that its functional dependence on wavelength is known and is described by just one parameter $\left(\kappa_{\lambda} \propto \lambda^{-\beta}\right)$. As Schnee et al. (2007) have shown, however, a three-wavelength constraint on the dust temperature $\left(T_{d}\right)$, column density $\left(N_{d}\right)$, and emissivity spectral index $(\beta)$ independently is only possible for the case of very (to date unrealistically) high signal-to-noise ratio $(\mathrm{S} / \mathrm{N})$ data. Nevertheless, with three wavelengths of very high $\mathrm{S} / \mathrm{N}$, or measurements at four or more wavelengths, one could constrain the temperature, column density, and emissivity independently, if only all the mixtures of grains along lines of sight were similar and the temperature distribution along the line of sight were isothermal.

In an earlier paper (Schnee et al. 2006, hereafter SBG06), we showed that line-of-sight variations in the dust temperature ac-

\footnotetext{
${ }^{1}$ Division of Physics, Mathematics and Astronomy, California Institute of Technology, 770 South Wilson Avenue, Pasadena, CA 91125; schnee@astro .caltech.edu.

${ }^{2}$ Harvard-Smithsonian Center for Astrophysics, 60 Garden Street, Cambridge, MA 02138
}

count for nearly all the scatter in a plot of the column density derived from dust emission versus that derived from dust extinction in the Perseus molecular cloud. In Schnee et al. (2005, hereafter SRGL05), we found that very small grains (VSGs) are likely to account for a large fraction ( $>50 \%)$ of the dust emission at wavelengths shorter than $100 \mu \mathrm{m}$. If that fraction is relatively constant, then one can say that big grains (BGs) are a good proxy for column density, modulo the uncertainty imposed by the lineof-sight variations in the dust temperature. However, if the VSG contribution is not constant, but depends on either $N_{d}$ or $T_{d}$, then the situation is further confounded. In this paper, we explore all of these possibilities, and we find, in agreement with earlier works (e.g., Stepnik et al. 2003), that the VSG contribution does seem to vary systematically with $T_{d}$ and $N_{d}$, and that it is important to account for this effect in estimates of dust column density and dust temperature.

\section{OBSERVATIONS}

SRGL05 showed that far-infrared emission maps can be used to determine the dust temperature and column density in molecular clouds once the conversion between the far-infrared (FIR) optical depth and the $V$-band extinction has been determined. This analysis took advantage of the IRIS (Improved Reprocessing of the IRAS Survey) maps at 60 and $100 \mu \mathrm{m}$ (MivilleDeschênes \& Lagache 2005) and a near-infrared (NIR) extinction map created from Two Micron All Sky Survey (2MASS) data as part of the COMPLETE (Coordinated Molecular Probe Line Extinction and Thermal Emission) Survey of Star-forming Regions (Ridge et al. 2006). Subsequently, SBG06 demonstrated that the column density determined from pairs of FIR emission maps should become more accurate and less biased when the emission is measured at longer wavelengths because of the decreased influence of line-of-sight temperature variations.

With the recent release of 70 and $160 \mu \mathrm{m}$ maps of Perseus taken with the Spitzer Space Telescope (Evans et al. 2003; Rebull et al. 2007), we can improve on the SRGL05 analysis. In this short paper, we combine the $100 \mu \mathrm{m}$ IRIS and $160 \mu \mathrm{m}$ Spitzer maps to derive dust temperature and column density maps that are not strongly biased by the emission from transiently heated VSGs. In addition, we are able to estimate the variation of the VSG emission and BG emissivity with $T_{d}$ and the $V$-band extinction $\left(A_{V}\right)$. 


\subsection{Spitzer}

We used the 70 and $160 \mu \mathrm{m}$ emission maps of the Perseus molecular cloud from the Spitzer Science Archive that were taken as part of the Spitzer Legacy program "From Molecular Cores to Planet-forming Disks" (c2d; Evans et al. 2003; Rebull et al. 2007). The map resolutions are $18^{\prime \prime}$ and $40^{\prime \prime}$ at 70 and $160 \mu \mathrm{m}$, respectively. The $\mathrm{c} 2 \mathrm{~d}$ delivery includes error maps, which account for systematic and random errors. The overall gain calibration uncertainty is $\sim 5 \%$ at $70 \mu \mathrm{m}$ (Gordon et al. 2007) and $\sim 12 \%$ at $160 \mu \mathrm{m}$ (Stansberry et al. 2007). The c $2 \mathrm{~d}$ error maps show that the random error for maps smoothed to $5^{\prime}$ resolution (the resolution of the extinction map) is $<1 \%$ at both 70 and $160 \mu \mathrm{m}$.

We applied color corrections to these archival fluxes, since they were determined under the assumption that the emission is from a blackbody at $10,000 \mathrm{~K}$, whereas we have assumed that the emission comes from a modified blackbody with an emissivity spectral index of $\beta=2$ (Draine \& Lee 1984) and an equilibrium temperature to be determined, but certainly less than $10,000 \mathrm{~K}$ (Schlegel et al. 1998). The color correction, which is combined with the dust temperature calculation, is further described in $\S 3.1$.

\subsection{IRAS/IRIS}

The IRIS flux density maps at 60 and $100 \mu \mathrm{m}$, in contrast with earlier releases of the IRAS all-sky maps, have been corrected for the effects of zodiacal dust and striping and have the proper gain and offset calibration (Miville-Deschênes \& Lagache 2005). The IRIS maps of Perseus have a resolution of approximately $4.3^{\prime}$ (although we smooth them to the $5^{\prime}$ resolution of our NIR extinction map for all analyses). The noise in the IRAS maps is approximately 0.03 and $0.06 \mathrm{MJy} \mathrm{sr}^{-1}$ at 60 and $100 \mu \mathrm{m}$, respectively (Miville-Deschênes \& Lagache 2005), which is $\lesssim 1 \%$ of the flux in the portion of Perseus included in the c $2 d$ survey. Like the Spitzer maps, these were color-corrected by us (following the IRAS Explanatory Supplement VI C.3). ${ }^{3}$ For consistency, we consider only the region of Perseus covered by the c2d MIPS maps at 70 and $160 \mu \mathrm{m}$ in this analysis.

\subsection{NIR Extinction Map}

An extinction map of Perseus based on the 2MASS pointsource catalog was created as part of the COMPLETE survey (Ridge et al. 2006) using the NICER algorithm, devised by Lombardi \& Alves (2001). This algorithm uses the near-infrared color excess of background stars to estimate the column density of foreground dust. When we directly compare NIR- and FIRderived column densities (e.g., Figs. 2, 4, and 9), all maps have been smoothed to a common resolution of $5^{\prime}$, since that is the resolution of the NICER extinction map of Perseus.

\section{ANALYSIS}

The flux density emitted by dust in thermal equilibrium is given by

$$
\begin{gathered}
S_{\nu}=\Omega B_{\nu}\left(T_{d}\right) N_{d} \alpha \nu^{\beta}, \\
B_{\nu}\left(T_{d}\right)=\frac{2 h \nu^{3}}{c^{2}} \frac{1}{\exp \left(h \nu / k T_{d}\right)-1} .
\end{gathered}
$$

In equation (1), $S_{\nu}$ is the observed flux density at frequency $\nu$, $\Omega$ is the solid angle of the beam, $B_{\nu}\left(T_{d}\right)$ is the blackbody emis-

\footnotetext{
${ }^{3}$ See http://cmbdata.gsfc.nasa.gov/product/iras/docs/exp.sup/ch6/C3.html.
}

sion from the dust at temperature $T_{d}, N_{d}$ is the column density of the dust, $\alpha$ is a constant that relates the flux density to the optical depth of the dust at frequency $\nu$, and $\beta$ is the emissivity spectral index of the dust, which we assume to be 2 throughout this paper (Draine \& Lee 1984). In SRGL05 we showed that $\beta=2$ is a reasonable value for the cold dust in Perseus.

Equation (1) is only true for BGs in thermal equilibrium and does not describe the emission from stochastically heated VSGs, which cannot be characterized by an equilibrium temperature (Draine \& Li 2001). The VSGs are expected to be a significant component of the observed emission in the IRAS $60 \mu \mathrm{m}$ and Spitzer $70 \mu \mathrm{m}$ bands, but they are not expected to contribute much in the IRAS $100 \mu \mathrm{m}$ and Spitzer $160 \mu \mathrm{m}$ bands ( Li \& Draine 2001). If we were to ignore the VSG emission, we would systematically overestimate the dust temperature and underestimate the column density in Perseus. We therefore account for it using a procedure that improves on that adopted by SRGL05, as described below.

\subsection{Dust Temperature}

The fluxes quoted in the IRAS and Spitzer emission maps are derived from the convolution of the total energy detected with the wavelength-dependent instrumental response and an assumed spectral energy distribution (SED) of the emitting source. The quoted flux density at wavelength $\lambda_{0}$ is related to the intrinsic flux density by (Stansberry et al. 2007)

$$
F_{\lambda_{0}}^{\text {quote }}=\frac{\int\left(F_{\lambda}^{\text {int }} / F_{\lambda_{0}}^{\text {int }}\right) \lambda R_{\lambda} d \lambda}{\int\left(F_{\lambda}^{\text {def }} / F_{\lambda_{0}}^{\text {def }}\right) \lambda R_{\lambda} d \lambda} F_{\lambda_{0}}^{\text {int }},
$$

where $F_{\lambda}^{\text {int }}$ is the true source SED, $F_{\lambda}^{\text {def }}$ is the default SED, and $R_{\lambda}$ is the instrumental response function (the sensitivity of the detector as a function of wavelength). The default SED for the Spitzer data is that of a 10,000 K blackbody, while the default SED for the IRIS data is $F_{\lambda} \propto 1 / \lambda$. We assume that the intrinsic SED is a modified blackbody with $\beta=2$ (Draine \& Lee 1984), so the color correction is temperature-dependent.

To derive the color correction and the dust temperature, we create a lookup table of the intrinsic flux density at $\lambda=60,70$, 100 , and $160 \mu \mathrm{m}$ for dust at temperatures between 5 and $100 \mathrm{~K}$, at $0.1 \mathrm{~K}$ intervals. We then calculate the flux density that would have been quoted at those wavelengths, using equation (3). Using this table, we are able to derive the color-corrected dust temperature from the ratio of the quoted flux densities, making the assumption that the dust emission is optically thin and isothermal along each line of sight. The IRAS $100 \mu \mathrm{m}$ and Spitzer $160 \mu \mathrm{m}$ maps are not significantly corrupted by VSG emission, so we derive a dust temperature map using our lookup table and the quoted value of $F_{100} / F_{160}$ after smoothing the emission maps to a common $\left(5^{\prime}\right)$ resolution.

The IRAS $60 \mu \mathrm{m}$ and Spitzer $70 \mu \mathrm{m}$ flux densities come from a combination of BG and VSG emission, but equations (1) and (3) are only accurate for BG emission. In SRGL05 we removed the VSG emission by assuming that a constant fraction of all of the $60 \mu \mathrm{m}$ emission can be attributed to VSGs. This proportion was found by minimizing the difference between the temperature maps derived from IRIS data and the dust temperature map derived by Schlegel et al. (1998), which was derived from DIRBE 100 and $240 \mu \mathrm{m}$ emission (and is therefore free from VSG contamination).

However, the proportion of 60 and $70 \mu \mathrm{m}$ flux emitted by VSGs is expected to vary with the environment. In regions with 
high extinction, the VSGs will be shielded from the interstellar radiation field (ISRF) and will therefore not be effectively heated. In cold, dense regions, dust grains may stick together, removing the VSG population. For instance, Stepnik et al. (2003) have shown that the VSG emission is greatly reduced in a dense filament in Taurus where $A_{V}>2.1$ and also found a corresponding increase in the dust emissivity. A similar transition region of altered dust composition has been reported using IRAS 60 and $100 \mu$ m emission maps (e.g., Laureijs et al. 1991, 1989; Boulanger et al. 1990).

Here we take advantage of the higher resolution of the IRAS $100 \mu \mathrm{m}$ and Spitzer $160 \mu \mathrm{m}$ images, as compared with the DIRBE maps, in order to remove the emission from VSGs that accounts for spatial variations in their contribution. We divide our dust temperature map, derived from the emission at 100 and $160 \mu \mathrm{m}$, into 10 equally populated temperature and extinction subsets (five temperature subsets for values of $A_{V}$ that are less than the median NIR-derived extinction, and five temperature subsets for higher values of $A_{V}$ ) and calculate the VSG contribution to the 60 and $70 \mu \mathrm{m}$ emission independently for each bin, using the dust temperature derived from the $100+160 \mu \mathrm{m}$ pair of emission maps. In each bin, we multiply the 60 and $70 \mu \mathrm{m}$ emission (smoothed to $5^{\prime}$ resolution) by the scaling factors $f_{60}$ and $f_{70}$, which are chosen such that the temperatures derived from $f_{60} F_{60} / F_{100}$ and $f_{70} F_{70} / F_{160}$ are best matched to the dust temperature derived from $F_{100} / F_{160}$. The comparatively low resolution of the DIRBE-derived temperature map prevented us from performing this more sophisticated correction in SRGL05. The scaling factors $\left(f_{60}\right.$ and $\left.f_{70}\right)$ used to account for the VSG emission are shown in Figure 1. Our assumptions that dust is isothermal along each line of sight and that the dust emissivity (characterized by $\alpha$ and $\beta$ in eq. [1]) is constant introduce uncertainties into our calculations. The effect of assuming isothermality is covered in SBG06, and we discuss the effects of variable dust emissivity in $\S 3.3$.

\subsection{Column Density: Constant Dust Emissivity}

Given the flux density at one wavelength and the dust temperature, the optical depth is given by

$$
\tau_{\lambda}=\frac{S_{\lambda}}{B_{\lambda}\left(T_{d}\right)},
$$

where $B_{\lambda}\left(T_{d}\right)$ is the Planck function and $S_{\lambda}$ is the observed flux density at wavelength $\lambda$.

The optical depth at wavelength $\lambda$ can be converted to the $V$-band extinction $\left(A_{V}\right)$ using the equation

$$
A_{V}=X_{\lambda} \tau_{\lambda}
$$

where $X_{\lambda}$ is the scale factor, dependent on the wavelength $(\lambda)$ of the emission map, that relates the thermal emission properties of the dust grains to their $V$-band absorption properties (eq. [5] from SRGL05). We solve for $X_{\lambda}$ for each pair of emission maps by minimizing the difference between the emission-derived column density (smoothed to the resolution of the extinction map) and the 2MASS absorption-derived column density, as in SRGL05 and SBG06. For the $60+100 \mu \mathrm{m}$ and $100+160 \mu \mathrm{m}$ pairs of emission maps, we derive the optical depth and the conversion factor $\left(\tau_{100}\right.$ and $\left.X_{100}\right)$ from the $100 \mu \mathrm{m}$ emission map. For the $70+160 \mu \mathrm{m}$ pair of emission maps, we derive $\tau_{160}$ and $X_{160}$ and scale both values in Figures 1 and 2 to the expected values at $100 \mu \mathrm{m}$, assuming $\beta=2$ and using the scale factor $\left(\lambda_{100} / \lambda_{160}\right)^{\beta}=0.39$.
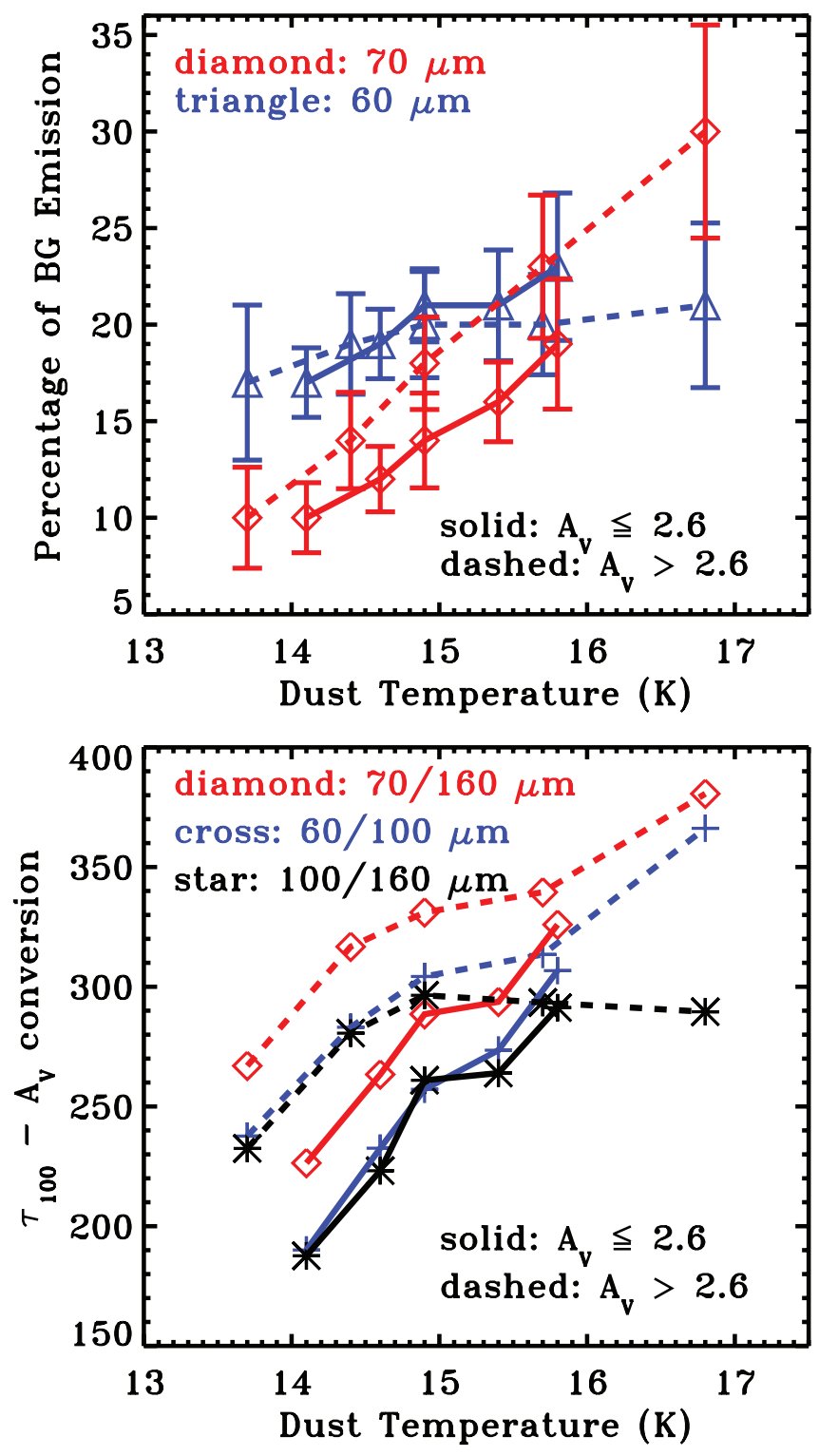

FIG. 1.-Top: Percentage of the observed 60 and $70 \mu \mathrm{m}$ flux emitted by BGs in thermal equilibrium as a function of dust temperature. The remaining portion is emitted by stochastically heated VSGs. The error bars show the standard deviation in each bin. Bottom: Ratio of the observed extinction to the $100 \mu \mathrm{m}$ optical depth as a function of dust temperature. For the $70+160 \mu \mathrm{m}$ pair of emission maps, we derive $\tau_{100}$ by assuming that $\beta=2$ and scaling $\tau_{160}$ by $(100 / 160)^{\beta}$. In both the top and bottom plots, there are $151 \pm 15$ independent $5^{\prime}$ data points in each bin.

The optimization sets the overall calibration for the FIR data, but it does not force the slope of the NIR-derived column density plotted against the FIR-derived column density to be exactly unity, although it will be close to this value. Variations in temperature along each line of sight will affect the emissionderived value of $A_{V}$ at high column density more than that at low column density, and the extinction-derived value of $A_{V}$ is unaffected by temperature fluctuations. This difference in the way the dust temperature is coupled to the derived column density, along with possible variations in the FIR dust emissivity, can change the slope of the emission- versus absorption-derived column density to be slightly different from unity. Note that equation (5) assumes that the dust emission and absorption properties are constant. The linear relationship between $\tau_{\mathrm{FIR}}$ and $A_{V}$ is shown in Figure 2 (left). 

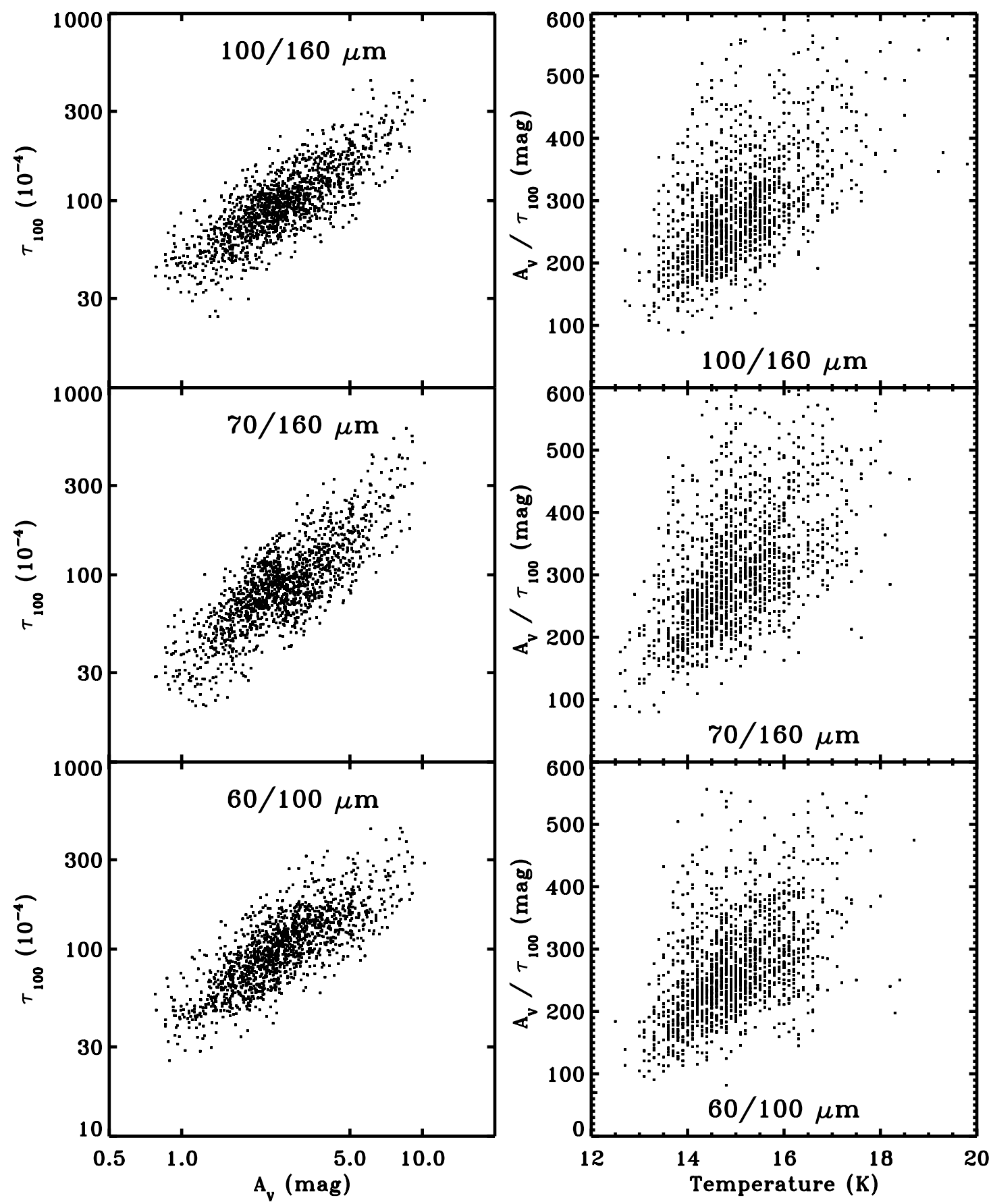

FIG. 2.-Left: Plots of the $100 \mu \mathrm{m}$ optical depth against the column density (expressed in terms of the $V$-band extinction) derived from the 2MASS map. Right: Ratio of the NIR-derived column density to the FIR-derived optical depth (at $100 \mu \mathrm{m}$ ), plotted against the FIR-derived dust temperature.

\subsection{Column Density: Variable Dust Emissivity}

Just as the emission from VSGs is expected to vary with the environment, so should the dust emissivity, due to such factors as grain growth through sticking and the formation of icy mantles. As a result of these processes, the dust emissivity in regions with higher column density and lower dust temperature is seen to be higher. This effect has been observed in many environments (e.g., Kiss et al. 2006; del Burgo \& Laureijs 2005; Bernard et al. 1999), where it has been shown that the FIR emissivity ( $\alpha$ in eq. [1], or $X_{\lambda}$ in eq. [5]) anticorrelates with dust temperature and varies by a factor of a few in interstellar clouds similar to Perseus. In Figure 2 (right) we show the FIR dust emissivity (expressed as the ratio of the $100 \mu \mathrm{m}$ optical depth and the NIR-derived extinction) plotted against the color temperature of the dust. We also find this dependence of the emissivity on temperature and column density, which suggests that the linear fit between $\tau_{\text {FIR }}$ and $A_{V}$ (see eq. [5] and Fig. 2, left) does not fully describe the relationship between dust emission and absorption.

It is important to note that temperature fluctuations along the line of sight can give the appearance of a temperature-dependent emissivity when the dust is assumed to be isothermal, even if the true dust emissivity is constant. Because the line-of-sight-averaged dust temperature is emission-weighted, the dust temperature is 

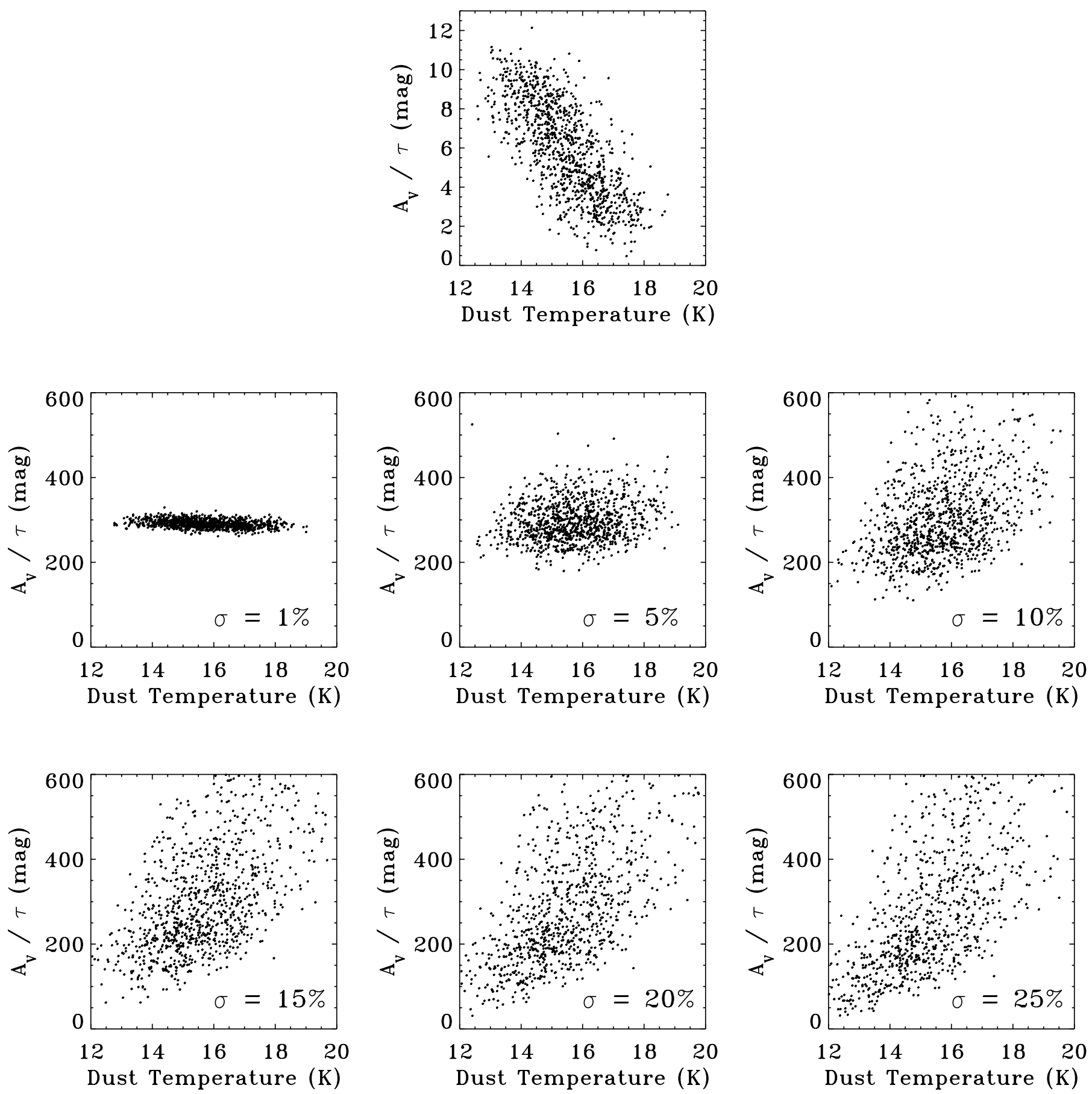

FIG. 3.- Top: Model temperature and column density distribution, which is used to generate synthetic emission maps and test the effects of noise on the derived dust

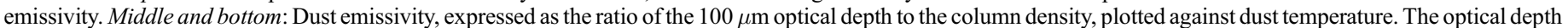

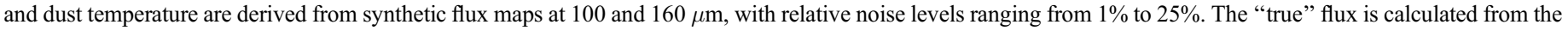

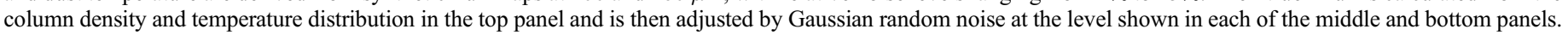

overestimated and the far-infrared optical depth is underestimated. This effect is larger along lines of sight with greater temperature variation (i.e., those passing through denser regions), which are preferentially colder than the more diffuse (and nearly isothermal) portions of the molecular cloud. As a consequence, the derived ratio of the far-infrared optical depth to extinction will be underestimated in the colder, denser regions of the molecular cloud. However, the observed temperature-dependent dust emissivity varies in the opposite direction of the correlation created by line-of-sight temperature fluctuations. The observed dust emissivity is larger at low temperatures, supporting the claim that the true dust emissivity is variable.

Noise in the FIR emission maps can also give the appearance of a variable dust emissivity. If noise changes the ratio of the fluxes, then the dust temperature will be either overestimated or underestimated. A high dust temperature will result in a low FIR-derived optical depth, and a low dust temperature will result in a high FIR optical depth. To illustrate the effect of noise on the derived dust emissivity, we analyze a simple model of a molecular cloud with constant dust emissivity in which the 

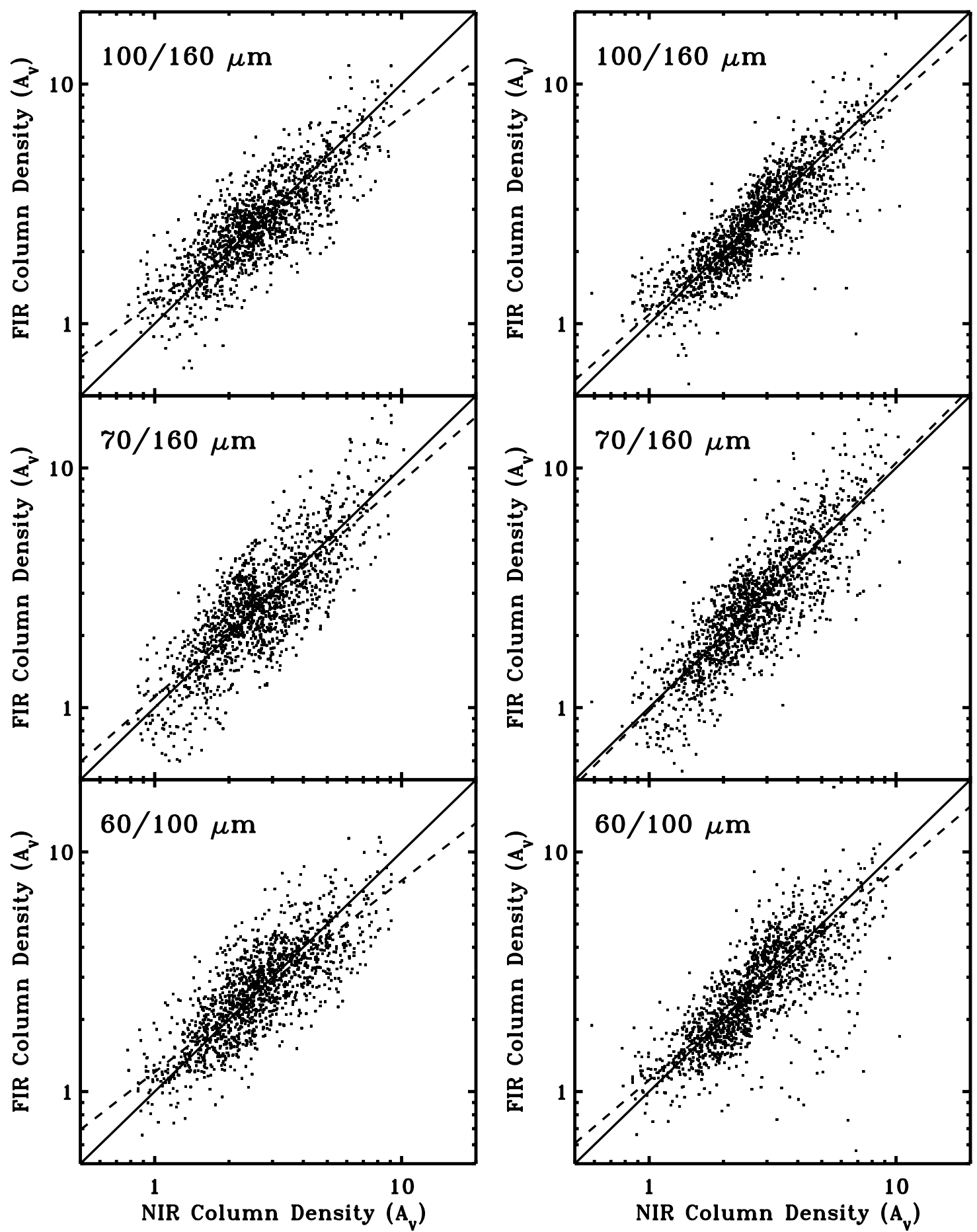

FIG. 4.-FIR-derived column density plotted against the NIR-derived column density. In the left panels, we assume a single value for the dust emissivity for each pair of emission maps, and in the right panels, we assume a variable value, as plotted in Fig. 1. [See the electronic edition of the Journal for a color version of this figure.]

temperature is anticorrelated with column density and where both quantities are within a typical range for molecular clouds. We then derive the flux at 100 and $160 \mu \mathrm{m}$ that would be emitted and add Gaussian random noise to the maps. We rederive the dust temperature and $100 \mu \mathrm{m}$ optical depth from the noisy maps and show the dust emissivity that would be estimated from the "observed" maps plotted against the temperature. The results are shown in Figure 3. The dust emissivity that we derive in Perseus (see Fig. 4) looks similar to what one would expect from maps of dust that have a constant emissivity, but that have noise on the level of $\sim 15 \%$. However, the random noise in all of our maps is much lower than this $(\lesssim 1 \%)$, so we can rule out the possibility that noise in the Perseus emission maps is tricking us into thinking that the dust emissivity is variable.

To account for the variable dust emissivity in the determination of the dust column density, we divide our data into the same 10 equally populated bins as we $\operatorname{did}$ in $\S 3.1$, on the basis of their NIR-derived extinction and temperature. Using equation (5), we derive the conversion $\left(X_{\lambda}\right)$ separately for each bin and each pair 
TABLE 1

Emission- versus Absorption-derived Column Density

\begin{tabular}{|c|c|c|c|c|c|c|}
\hline Emission Maps & $\begin{array}{c}\mu^{\mathrm{a}} \\
\text { Constant }\end{array}$ & $\begin{array}{c}\mu^{\mathrm{b}} \\
\text { Variable }\end{array}$ & $\begin{array}{c}\sigma^{\mathrm{c}} \\
\text { Constant }\end{array}$ & $\begin{array}{c}\sigma^{\mathrm{d}} \\
\text { Variable }\end{array}$ & $\begin{array}{c}\text { Slope }^{\mathrm{e}} \\
\text { Constant }\end{array}$ & $\begin{array}{c}\text { Slope }^{\mathrm{f}} \\
\text { Variable }\end{array}$ \\
\hline $60+100 \mu \mathrm{m}$ & 1.01 & 1.00 & 0.32 & 0.31 & 0.80 & 0.88 \\
\hline $70+160 \mu \mathrm{m}$ & 1.00 & 1.00 & 0.34 & 0.32 & 0.90 & 1.03 \\
\hline $100+160 \mu \mathrm{m}$ & 1.00 & 1.00 & 0.30 & 0.25 & 0.77 & 0.91 \\
\hline
\end{tabular}

${ }^{a}$ Median ratio of $A_{V, \mathrm{em}} / A_{V \text {, ext }}$, assuming a constant dust emissivity.

b Median ratio of $A_{V, \mathrm{em}} / A_{V \text {, ext }}$, assuming a variable dust emissivity.

c $1 \sigma$ scatter in $A_{V, \mathrm{em}} / A_{V \text {,ext }}$, assuming a constant dust emissivity.

d $1 \sigma$ scatter in $A_{V \text {, em }} / A_{V \text {, ext }}$, assuming a variable dust emissivity.

e Best-fit slope from Fig. 4 (left).

${ }^{\mathrm{f}}$ Best-fit slope from Fig. 4 (right).

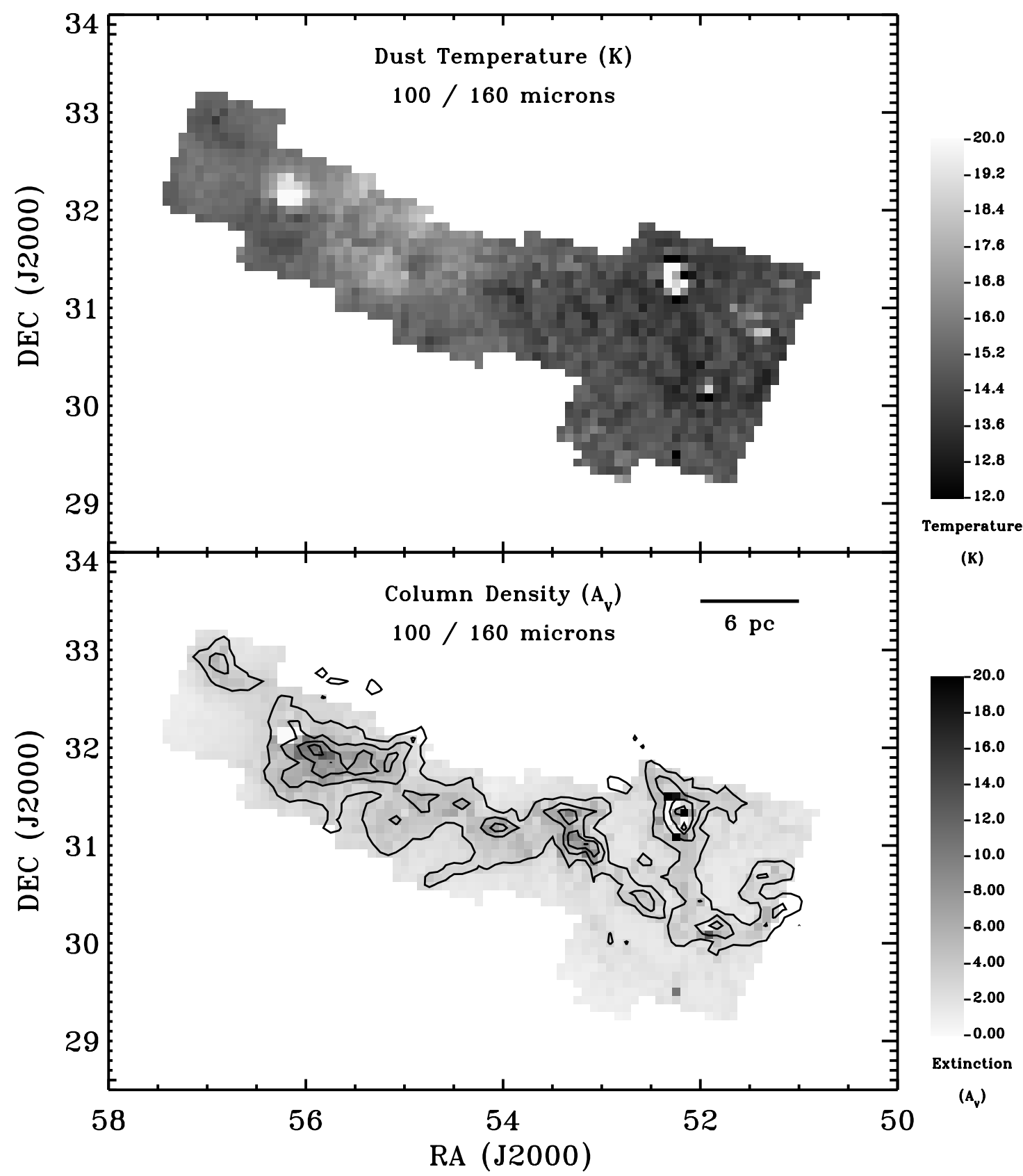

FIG. 5.-Dust temperature map (top) and column density (expressed in terms of $V$-band extinction; bottom) derived from the IRAS $100 \mu \mathrm{m}$ and Spitzer $160 \mu \mathrm{m}$ flux density maps, at the $5^{\prime}$ resolution of our NIR-derived extinction map. The column density is derived with a variable dust emissivity. Black contours in the bottom panel show the regions with extinctions of $A_{V}=3,5,7$, and 9. [See the electronic edition of the Journal for a color version of this figure.] 


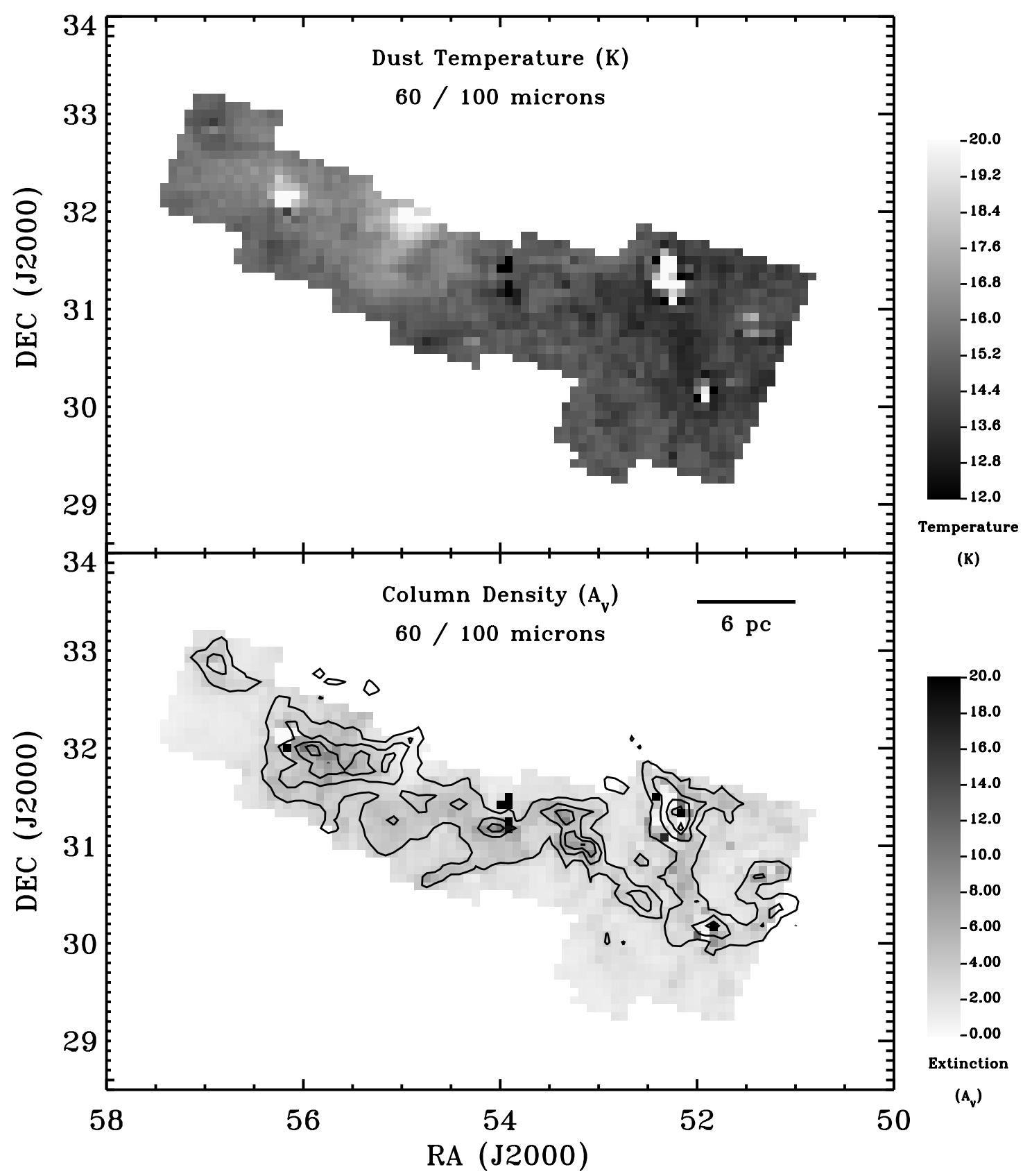

FIG. 6.-Same as Fig. 5, but for values derived from the IRAS 60 and $100 \mu \mathrm{m}$ flux density maps. [See the electronic edition of the Journal for a color version of this figure.]

of emission maps. The derived dust emissivity, as a function of $T_{d}$, is plotted in Figure 1.

\section{RESULTS}

\subsection{VSG Emission and Dust Emissivity versus $T_{d}$ and $A_{V}$}

As is shown in Figure 1, the 60 and $70 \mu \mathrm{m}$ emission is dominated by the emission from VSGs. The fractional contribution of BG emission rises with increasing $T_{d}$ at both 60 and $70 \mu \mathrm{m}$. This is no surprise, given that warmer dust will have larger $60+100$ and $70+160 \mu \mathrm{m}$ flux ratios. At $70 \mu \mathrm{m}$, the BG contribution is a larger percentage of the total flux for $A_{V}>2.6$ than $A_{V}<2.6$, while at $60 \mu \mathrm{m}$ the difference is smaller and changes sign. This difference may be caused by the 60 and $70 \mu \mathrm{m}$ passbands, since $I R A S$ is sensitive (sensitivity $\geq 10 \%$ ) from 37 to $82 \mu \mathrm{m}$, whereas Spitzer is sensitive from $5 \overline{5}$ to $86 \mu \mathrm{m}$.
The parameter used to derive the dust column density from the FIR optical depth of the dust, as given by $X_{\lambda}$ in equation (5), varies nearly identically with $T_{d}$ for all three pairs of emission maps. The evolution in $X_{\lambda}$ is primarily caused by the variable emissivity of the dust (shown in Fig. 2), but also by line-of-sight temperature fluctuations and noise in the emission maps. Taking into account variations in the VSG contribution to emission from dust with $\lambda<100 \mu \mathrm{m}$ and variations in the emissivity of the BGs represents an increase in the complexity of the analysis performed in SRGL05.

\subsection{Emission versus Absorption-derived Column Density}

As discussed in SRGL05, the point-to-point scatter between the emission- and absorption-derived column densities can be quite large (a few tens of percent). This scatter can be attributed to lineof-sight temperature fluctuations, nonconstant dust emissivity, 


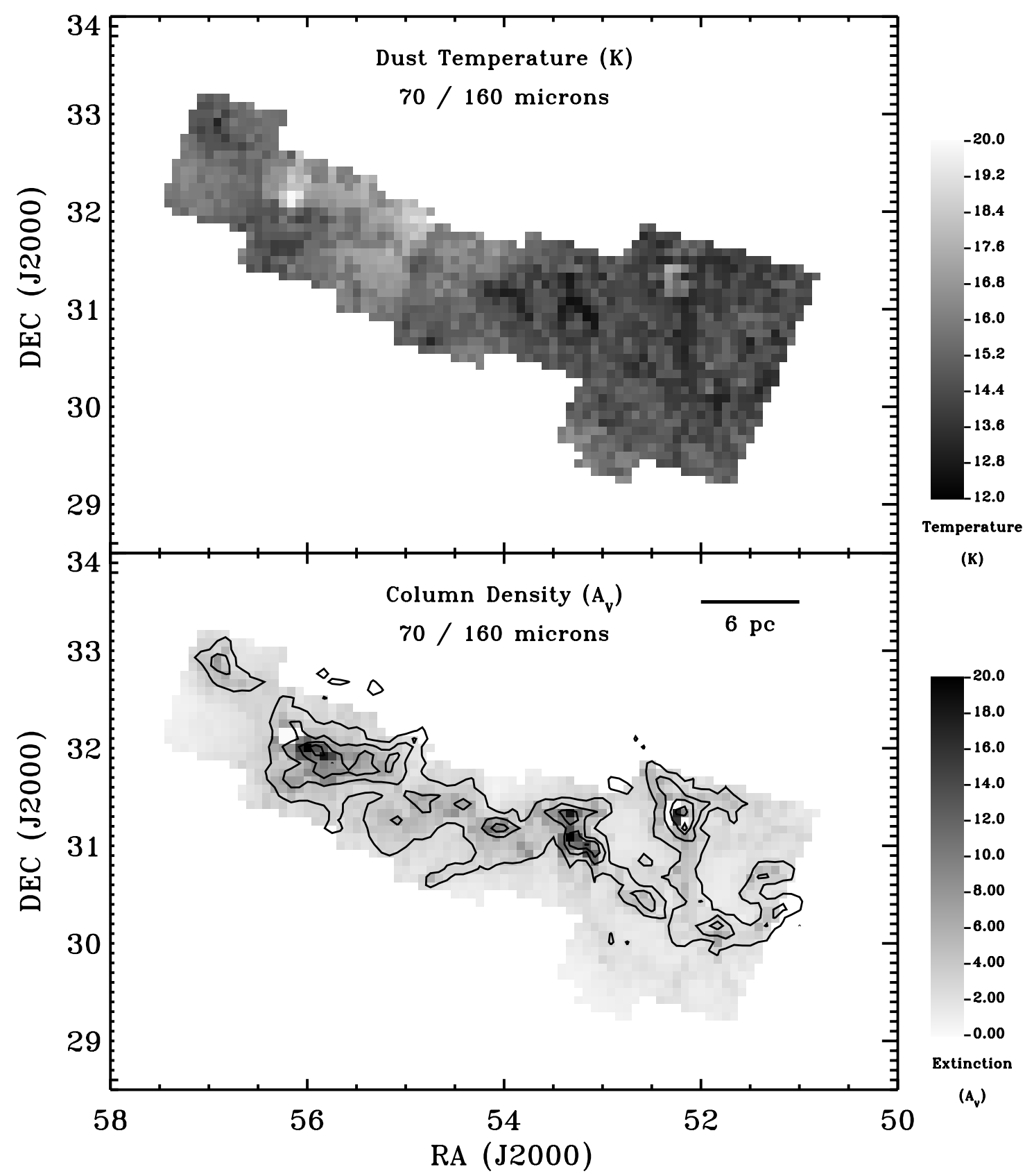

Fig. 7.-Same as Fig. 5, but for values derived from the Spitzer 70 and $160 \mu \mathrm{m}$ flux density maps. [See the electronic edition of the Journal for a color version of this figure.]

and variations in the fraction of emission coming from VSGs (although this last source of error should not affect the column density derived from emission maps at or above $\sim 100 \mu \mathrm{m}$ ).

In this paper we calculate the emission-derived column density in two ways. In the first method we assume that the dust emissivity is constant in Perseus and that $A_{V}$ scales with the FIR optical depth (eq. [5]). Our second method uses a dust emissivity that varies with $A_{V}$ and $T_{d}$, as shown in Figure 1 . In both cases we correct for variations in the relative VSG/BG contribution to the 60 and $70 \mu \mathrm{m}$ emission, as a function of $T_{d}$ and $A_{V}$, as described in $\S 3.1$. The values for the median column density ratio, the scatter around that median, and the slope of the best-fit line between the emission- versus absorption-derived column densities for Perseus are shown in Table 1, and the corresponding plots are shown in Figure 4.
In SBG06, we use a simplified model of emission from an externally heated molecular cloud in order to study the effects of line-of-sight temperature variations on column density estimates without having to worry about the effects of VSG emission or variations in the dust emissivity. Using that model, we predict that the scatter and bias should be largest for the $60+100 \mu \mathrm{m}$ pair of maps and smallest for the $100+160 \mu \mathrm{m}$ pair. However, in this paper, when we use a single $\tau-A_{V}$ conversion, we find that the slope and scatter between our emission- and absorptionderived column densities are very similar for all three pairs of emission maps, in conflict with SBG06. When the column density map derived from NIR extinction is plotted against that derived from dust emission at 60 and $100 \mu \mathrm{m}$, the scatter is consistent with the prediction from the model in SBG06. The larger than expected scatter seen in the $70+160 \mu \mathrm{m}$ pair of maps is 


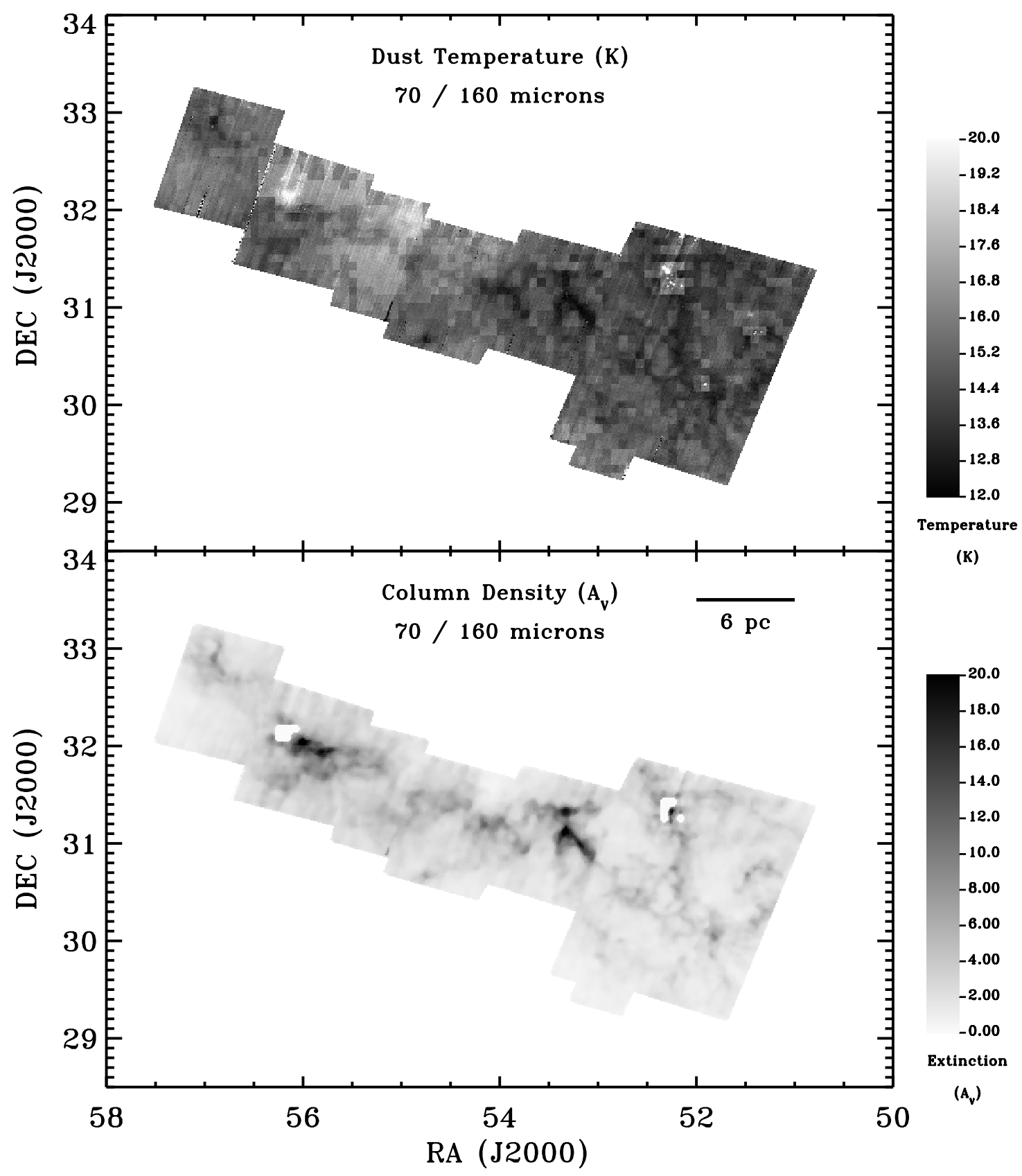

Fig. 8. - Similar to Fig. 7, but for values at the $40^{\prime \prime}$ resolution of the $160 \mu \mathrm{m}$ map. [See the electronic edition of the Journal for a color version of this figure.]

probably not caused by an improper VSG correction, since we also find excess scatter in the NIR-derived column density versus that derived from the $100+160 \mu \mathrm{m}$ maps, and VSG emission should be insignificant in the 100 and $160 \mu \mathrm{m}$ maps.

When we divide the emission maps into 10 subsets and derive the $\tau-A_{V}$ conversion for each bin (shown in Fig. 1), there is less bias in the emission-derived column density. This is shown by the fit between the FIR- and NIR-derived column densities in Figure 4 , which has a slope of $\sim 1$. However, the scatter in the relation (see Table 1) is only significantly improved for the column densities derived from the $100+160 \mu \mathrm{m}$ maps compared to the constant $\tau-A_{V}$ derivation. This suggests that the emission properties of dust are more complicated than those assumed in this paper. For instance, the emissivity (or spectral index) of the dust could be density-dependent, or there could be variations in the grain properties as a function of position in Perseus, and not merely as a function of dust temperature and column density.
We conclude that the $100+160 \mu \mathrm{m}$ pair of maps is the best for deriving dust temperature and column density because it is least affected by the emission from VSGs and has the least NIR versus FIR column density scatter. However, since the 70+160 pair of emission maps has much better resolution, the column density and dust temperature maps produced from it are also useful if one is willing to accept the additional uncertainty of using maps presented at $\sim 40^{\prime \prime}$ that have been calibrated on a $\sim 5^{\prime}$ scale.

\subsection{Temperature and Column Density Maps}

Here we present dust temperature and column density maps derived from the IRAS and Spitzer fluxes. Figures 5, 6, and 7 show the temperature and column density maps derived from the $I R A S$ and Spitzer far-infrared data and are presented at $\sim 5^{\prime}$ resolution (which is the resolution of the NIR-derived extinction map and is approximately the resolution of the IRIS 60 and $100 \mu \mathrm{m}$ 

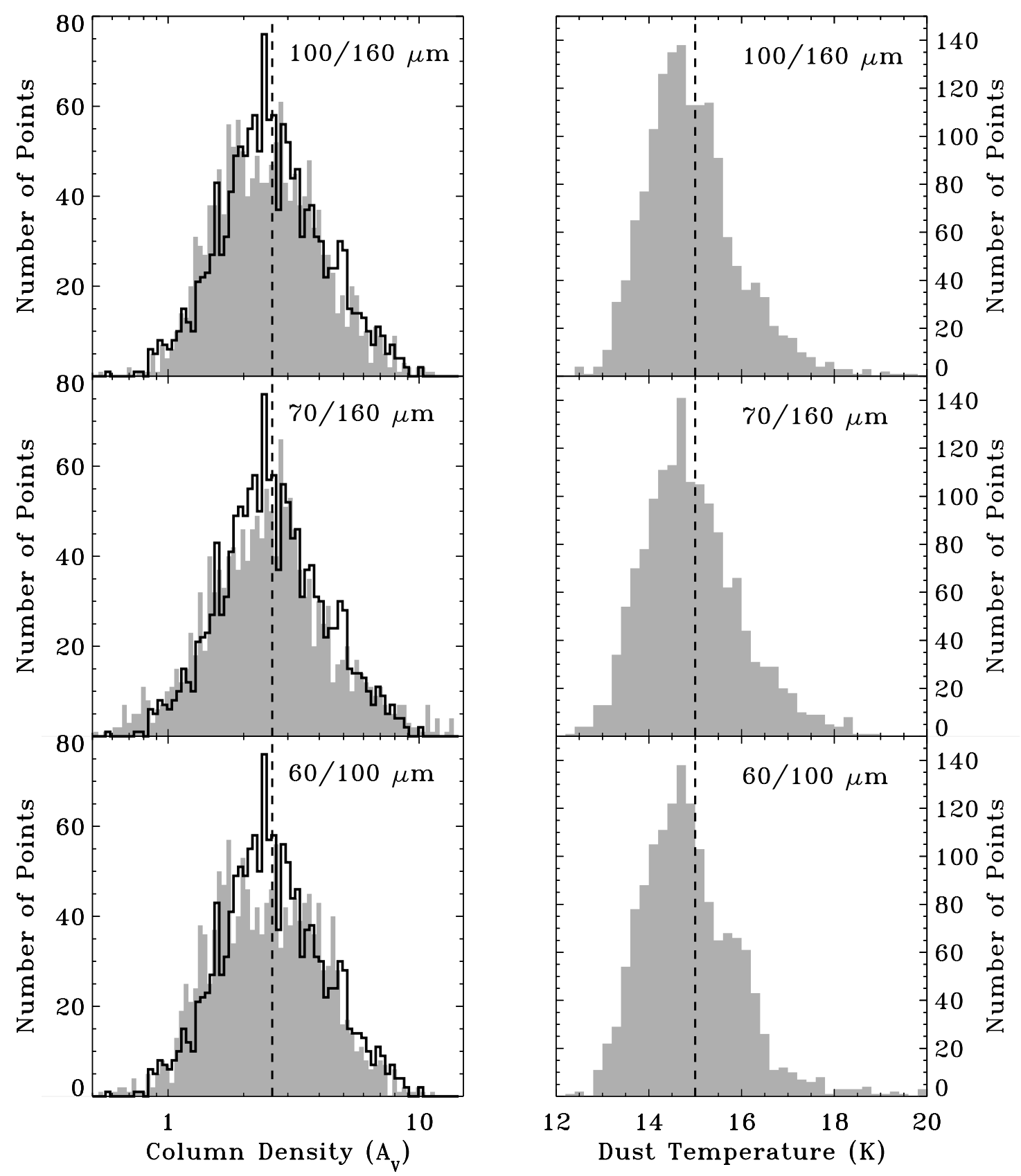

FIG. 9.- Histograms of the dust color temperature and column density derived from the IRAS 60 and $100 \mu \mathrm{m}$ flux density maps (bottom), the Spitzer 70 and $160 \mu \mathrm{m}$ flux density maps (middle), and the IRAS $100 \mu \mathrm{m}$ and Spitzer $160 \mu \mathrm{m}$ flux density maps (top). The column density is derived with a variable dust emissivity. The dust temperature is derived with a variable fraction of the 60 and $70 \mu \mathrm{m}$ flux emitted by BGs. The filled histograms show the column density and temperature derived from FIR emission, whereas the open histograms show the column density derived from NIR absorption. The vertical dashed lines show the median value of $A_{V}$ in the $2 \mathrm{MASS}$ map (left) and the median dust temperature in the $100+160 \mu \mathrm{m} T_{d}$ map (right). [See the electronic edition of the Journal for a color version of this figure.]

maps). Figure 8 is derived from the Spitzer 70 and $160 \mu \mathrm{m}$ data and is presented at the $40^{\prime \prime}$ resolution of the $160 \mu \mathrm{m}$ map (the $70 \mu \mathrm{m}$ emission map was smoothed to the resolution of the $160 \mu \mathrm{m}$ map). The column densities are determined by assuming a variable dust emissivity, as described in $\S 3.3$. Note that the VSG subtraction and variable dust emissivity are calibrated at $5^{\prime}$ resolution, so the calibration of the dust temperature and column density using the Spitzer 70 and $160 \mu \mathrm{m}$ maps presented at $40^{\prime \prime}$ resolution is more uncertain than the maps presented at $5^{\prime}$ resolution.

\subsection{Temperature and Column Density Histograms}

Figure 9 shows histograms of the dust temperature and column density distributions derived from the pairs of emission maps at
$60+100,70+160$, and $100+160 \mu \mathrm{m}$. The 60 and $70 \mu \mathrm{m}$ fluxes have been scaled to remove the VSG emission (as explained in $\S 3.1$ ). The median temperature derived for this region of the Perseus molecular cloud by Schlegel et al. (1998) is $16.5 \mathrm{~K}$, whereas we derive a median temperature of $15.0 \mathrm{~K}$, so our derived temperature is about $10 \%$ lower. The emission-derived column density is scaled to match that derived from the NIR reddening of background stars. Therefore, it is no surprise that the $A_{V}$ distributions in all three rows of Figure 9 are similar to that of the extinction map.

\section{CONCLUSION}

Using Spitzer 70 and $160 \mu \mathrm{m}$ maps released by the c $2 \mathrm{~d}$ Legacy Project, we have created high-resolution dust temperature and 
column density maps of the Perseus molecular cloud. Although they are smaller in area than similar maps presented in SRGL05 using IRAS and 2MASS data, the maps in this paper (Fig. 7) are better calibrated to account for variations in the dust emissivity and the removal of VSG emission. Combining the Spitzer $160 \mu \mathrm{m}$ map with the IRAS $100 \mu \mathrm{m}$ map, we show that the dust temperature in Perseus is significantly colder (by $\sim 10 \%$ ) than reported in the dust temperature map derived by Schlegel et al. (1998).

We find that the dust emissivity varies with $A_{V}$ and $T_{d}$, in agreement with the results of del Burgo \& Laureijs (2005) and Kiss et al. (2006). By fitting the absorption-derived column density to the FIR opacity in each of 10 subsets of the data, we demonstrate an improved method for calculating the emission-derived dust column density. The column density derived from the $60+$ $100 \mu \mathrm{m}$ pair of emission maps, when compared with the NIR $A_{V}$, has a slope and a scatter consistent with those expected from variations in temperature along the line of sight. However, the scatter between the absorption-derived and the 70+160 and 100+160 $\mu \mathrm{m}$ emission-derived column densities is larger than would be expected from line-of-sight variations in the dust temperature alone. One possible explanation for the large scatter is that the $160 \mu \mathrm{m}$ map is more sensitive to the emission from cold dust grains than are the 60 and $100 \mu \mathrm{m}$ maps (SBG06), and the dust emissivity varies more strongly at colder $\left(T_{d}<15 \mathrm{~K}\right)$ temperatures. Although the origin of the observed temperature dependence is uncertain, recent theoretical models of amorphous dust grains by Meny et al. (2007) and Boudet et al. (2005) also exhibit an anticorrelation between dust temperature and emissivity. Another possible cause for the higher dust emissivity at lower temperatures is that the dust grains could be accreting icy mantles in the dense and colder regions within the molecular cloud. Regardless of the reason, it is not surprising that dust grains with a higher emissivity, given their ability to cool more efficiently, will be found at lower equilibrium temperatures.

A temperature-dependent dust emissivity presents a challenge for those attempting to derive dust temperatures and column densities from FIR emission maps. In SBG06, we suggest that longer wavelength $(\lambda>100 \mu \mathrm{m})$ emission maps should be used to calculate the line-of-sight dust properties because of their decreased sensitivity to fluctuations in the dust temperature and the lack of confusing emission from VSGs. In this paper, we show that the dust emissivity anticorrelates with dust temperature and that the variability is likely to increase at lower temperatures, an effect that is also seen in other regions (e.g., del Burgo \& Laureijs 2005; Kiss et al. 2006). This complicates the interpretation of longer wavelength emission maps, which are more sensitive to cold dust. The deleterious effects of VSG emission, temperature gradients, and variable dust emission properties add complexity to the determination of the mass and temperature distributions of dust in a molecular cloud. Resolution of these problems may require coverage from tens to hundreds of microns, finer spectral resolution than the broad IRAS and Spitzer filters provide, and comparison with dust emission models of "realistic" molecular clouds.

Given the complexity of determining the column density from dust emission, one may wonder if using extinction might be a superior method. For instance, the extinction map of Perseus made with 2MASS data and the NICER algorithm (Ridge et al. 2006; Lombardi \& Alves 2001) is used as a template with which we calibrate the emission-derived column density in this paper, and we trust extinction-derived column density more than that derived from dust emission in general (Goodman et al. 2008; Pineda et al. 2008). The resolution of extinction maps is limited by the number of background tracers with known color, which provide $1^{\prime}$ resolution for clouds nearby the Galactic center in projection (Lombardi et al. 2006), but is coarser toward clouds further away from the Galactic plane. However, emission maps are limited only by the size of the detector and also provide information on the dust temperature.

We would like to thank our anonymous referee for substantially improving the scope and clarity of this paper. Scott Schnee acknowledges support from the Owens Valley Radio Observatory, which is supported by the National Science Foundation through grant AST 05-40399. Alyssa Goodman acknowledges support from the National Science Foundation under grant AST-0407172.

\section{REFERENCES}

Bernard, J. P., et al. 1999, A\&A, 347, 640

Boudet, N., Mutschke, H., Nayral, C., Jäger, C., Bernard, J.-P., Henning, T., \& Meny, C. 2005, ApJ, 633, 272

Boulanger, F., Falgarone, E., Puget, J. L., \& Helou, G. 1990, ApJ, 364, 136

del Burgo, C., \& Laureijs, R. J. 2005, MNRAS, 360, 901

Draine, B. T., \& Lee, H. M. 1984, ApJ, 285, 89

Draine, B. T., \& Li, A. 2001, ApJ, 551, 807

Evans, N. J., II, et al. 2003, PASP, 115, 965

Goodman, A. A., Pineda, J. E., \& Schnee, S. L. 2008, ApJ, submitted (arXiv:0806.3441)

Gordon, K. D., et al. 2007, PASP, 119, 1019

Kiss, C., Ábrahám, P., Laureijs, R. J., Moór, A., \& Birkmann, S. M. 2006, MNRAS, 373, 1213

Laureijs, R. J., Chlewicki, G., Wesselius, P. R., \& Clark, F. O. 1989, A\&A, 220, 226

Laureijs, R. J., Clark, F. O., \& Prusti, T. 1991, ApJ, 372, 185

Li, A., \& Draine, B. T. 2001, ApJ, 554, 778
Lombardi, M., \& Alves, J. 2001, A\&A, 377, 1023

Lombardi, M., Alves, J., \& Lada, C. J. 2006, A\&A, 454, 781

Meny, C., Gromov, V., Boudet, N., Bernard, J.-Ph., Paradis, D., \& Nayral, C. 2007, A\&A, 468, 171

Miville-Deschênes, M.-A., \& Lagache, G. 2005, ApJS, 157, 302

Pineda, J. E., Caselli, P., \& Goodman, A. A. 2008, ApJ, 679, 481

Rebull, L. M., et al. 2007, ApJS, 171, 447

Ridge, N. A., et al. 2006, AJ, 131, 2921

Schlegel, D. J., Finkbeiner, D. P., \& Davis, M. 1998, ApJ, 500, 525

Schnee, S., Bethell, T., \& Goodman, A. 2006, ApJ, 640, L47 (SBG06)

Schnee, S., Kauffmann, J., Goodman, A., \& Bertoldi, F. 2007, ApJ, 657, 838

Schnee, S. L., Ridge, N. A., Goodman, A. A., \& Li, J. G. 2005, ApJ, 634, 442 (SRGL05)

Stansberry, J. A., et al. 2007, PASP, 119, 1038

Stepnik, B., et al. 2003, A\&A, 398, 551

Wood, D. O. S., Myers, P. C., \& Daugherty, D. A. 1994, ApJS, 95, 457 